\title{
LA DIMENSIÓN INTERNA DE LA RESPONSABILIDAD SOCIAL
}

\author{
ROSSANA GRECO \\ MENCIÓN ESPECIAL DEL JURADO \\ CONCURSO $60^{\circ}$ ANIVERSARIO \\ RESPONSABILIDAD SOCIAL UNIVERSITARIA.
}

FACULTAD DE CIENCIAS ECONÓMICAS - UNNE 
"La Responsabilidad Social tiene la característica de funcionar como un bumerang, que vuelve con ímpetu al que lo lanza" (Cammarota y Berenblum, 2009, p.61).

\title{
INTRODUCCION
}

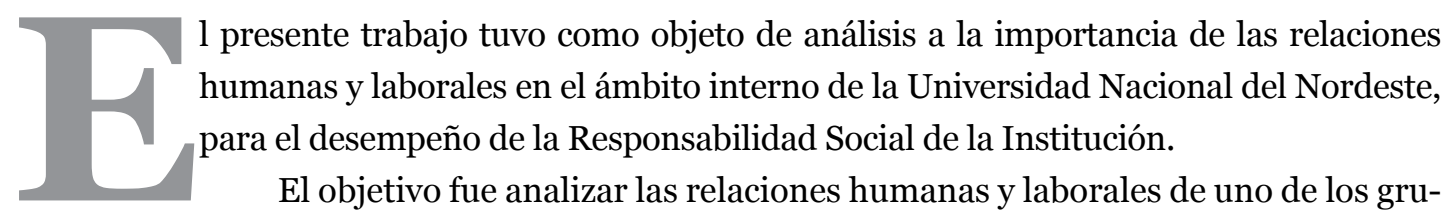
pos de interés -formado por el personal docente y no docente- como una de las dimensiones necesarias para optimizar el desempeño en Responsabilidad Social de la Universidad.

La metodología seguida fue de tipo bibliográfico, siguiendo, principalmente, autores como Bernardo Kliskberg, Francois Vallaeys, Federico Saravia y Héctor Larocca.

Del análisis efectuado se concluye que la dimensión de gestión interna debe ser periódicamente revisada y evaluada, fomentando una comunicación fluída y continua con el grupo de interés seleccionado para este trabajo.

Palabras clave: Responsabilidad Social Universitaria - Grupos de interés - Calidad de vida.

\begin{abstract}
This study was analyzed the importance of human relations and labor in the domestic sphere of the Universidad Nacional del Nordeste, to fulfill the social responsibility of the institution.

The aim was to analyze human relationships and work of one of the groups of interest formed by the teachers and non-teaching staff as a necessary dimension to optimize performance in Social Responsibility of the University.

The methodology was bibliographical, following mainly authors such as Bernardo Kliksberg, Francois Vallaeys, Federico Saravia and Hector Federico Larocca.

Of the analysis we conclude that the dimension of internal management should be periodically reviewed and evaluated, fostering a continuous fluid communication with the interest group selected for this work.
\end{abstract}

Keywords: University Social Responsibility - Groups of Interest - Quality of life. 


\section{INTRODUCCIÓN}

El presente trabajo tiene como objeto de análisis a las relaciones humanas y laborales, de uno de los grupos de interés de la Universidad Nacional del Nordeste, formado por su personal docente y no docente, para el desempeño de la Responsabilidad Social de la Institución.

El objetivo fue analizar las relaciones humanas y laborales de este grupo de individuos, como una de las dimensiones necesarias para optimizar el nivel de desempeño en acciones de Responsabilidad Social de la Universidad.

La metodología seguida fue de tipo bibliográfico, siguiendo autores como Bernardo Kliskberg, quien expresa que "las Universidades tienen un compromiso ético en su accionar y no solo deben contemplar el aspecto técnico y tecnológico; que la Responsabilidad Social prestigia a la Organización, atrae a los mejores talentos, mejora la competitividad y sustentabilidad"; Francois Vallaeys (s.f.), por su parte, opina que la Responsabilidad Social se refiere a un modo de comprender la ética diferente del habitual y que está en práctica la ética de la acción y de la responsabilidad por las consecuencias inmediatas y a futuro de la acción, y no de la intención que prioriza los motivos por las que se realiza la acción. Federico Saravia (2009) manifiesta que la responsabilidad social exige una toma de conciencia respecto del lugar que ocupan en la escala social aquellos que están llamados a asumirla, por su desempeño, en contextos organizacionales; ya sea el individuo dentro de la organización, como el lugar de la organización misma en sus distintos contextos...Cuando este compromiso es asumido y se manifiesta en hechos concretos, estas acciones contribuyen a mejorar la calidad de vida promedio de la comunidad de referencia... afirmando la centralidad de la responsabilidad social que permite pensarla como una forma o un estilo en que se efectiviza esa actividad, atravesando la gestión y se verifica en el conjunto de decisiones de la vida organizacional”. En esta línea, Héctor Larocca (2009) sostiene que la Responsabilidad Social tiene una fuerte impronta pragmática y que diseñar los dispositivos adecuados para canalizar las acciones y evaluar sus impactos en el conjunto de los stakeholders, le agrega la dimensión de sustentabilidad o sea permanencia en el tiempo.

\section{LA RESPONSABILIDAD SOCIAL UNIVERSITARIA}

La Responsabilidad Social promueve una nueva cultura que cuestiona la distorsión de los valores, proponiendo a la ética como base de una gestión transparente y participativa, resaltando la importancia del respeto entre los hombres y por la naturaleza, el diálogo entre los grupos interesados y afectados por su actividad y la creación de valor compartido en sus relaciones.

Las relaciones con creación de valor compartido, implica que todos deben beneficiarse, en un plano de igualdad, aportando a un desarrollo sano de todos los participantes, en pos de una mejor calidad de vida.

Una organización es responsable ante todos aquellos individuos o grupos de individuos, conocidos como grupos de interés (en inglés stakeholders), que se encuentran implicados o

${ }_{1}^{1}$ Video de inicio del Programa Iberoamericano de Formación en Formadores de Responsabilidad Social Empresaria de las Naciones Unidas para el Desarrollo. En: http://www.youtube.com/watch?v=WyEaS7Q7E8E\&feature=endscreen\&NR=1 
involucrados en su actividad, entre los que se pueden mencionar a los empleados, directivos, proveedores, gobierno, competidores, comunidad, entre otros.

Los stakeholders "son aquellos para los que la organización es significativamente responsable sobre su bienestar, o mantienen un reclamo moral o legal en la organización” (Langtry,1994,p.58).

La Responsabilidad Social no es caridad ni filantropía. Si bien estas actitudes tienen en común que implican acciones buenas, se diferencian porque una conducta responsable se mide por los efectos inmediatos y colaterales que produce, y no solo por hacer acciones buenas en sí mismas sin medir las consecuencias e impactos que ocasiona en su contexto de actuación.

"La Responsabilidad Social debe: primero sentirse; luego pensarse; más tarde expresarla; y por último hacerla...asumirla ligeramente la predestina a ser considerada una moda" (Larroca, 2009, p. 155).

La Responsabilidad Social promueve una cultura del reporte, incentivando la comunicación -interna y externa- de sus acciones, la evaluación de sus impactos y rediseño de sus políticas, en un proceso de mejora continua, para reducir las consecuencias negativas.

En el desarrollo de la actuación socialmente responsable de las organizaciones, una de las tareas más complejas es crear relaciones de confianza con los stakeholders. Para ello cada ente debe identificar y definir sus grupos interesados, para que sus expectativas puedan ser contempladas e incorporadas a su planificación estratégica, como así también, se deben desarrollar canales para mantener una comunicación fluida y continua.

\section{La importancia de los stakeholders es de}

tal magnitud, que la reputación de una

Organización depende de las percepciones

que tienen dichos grupos de ella.

La Responsabilidad Social en la Universidad se presenta como una nueva forma de gestión basada en la coherencia que debe existir entre los fines para la cual fue creada y las acciones concretas que realiza.

En este escenario de las Instituciones de Nivel Superior, el reconocimiento que éstas poseen en la comunidad, les exige un alto nivel de compromiso social. Esto conlleva hablar hoy de Responsabilidad Social Universitaria -RSU- como un tipo especial de Responsabilidad con identidad propia.

La RSU se entiende en términos de la Responsabilidad de todos los que la forman; solo con esta mirada podemos alejarnos de lo abstracto, para centrarnos en algo concreto, traducido en acciones. No podemos pensar en una organización socialmente responsable, con integrantes ajenos a conductas éticas y sin sentido de pertenencia. Este escenario nos lleva a profundizar en este aspecto.

La influencia que tiene hoy la Universidad en la comunidad sirve de base para que se produzca un efecto enraizante, es decir, la Universidad se posiciona y se arraiga en la medida 
que genere conciencia individual de la importancia que tiene cada uno de sus miembros en comportarse responsablemente.

\section{La Responsabilidad Social de la Universidad se apalanca en la responsabilidad de quienes la integran, y así se potencian las acciones en ese sentido.}

Esta afirmación debe ser conocida por el personal docente y no docente de la Universidad, para generar conciencia de la importancia que tiene cada uno en su puesto de trabajo y de los aportes que, desde su lugar, brinda. Para ello es imperioso que el docente dimensione el poder que tiene en el prestigio de la Institución y de influir éticamente, en forma directa, sobre los estudiantes, orientándolos en su accionar hacia un comportamiento solidario, responsable y comprometido; que cada trabajador no docente reconozca el valor de cada una de las tareas que realiza y de cuánto aporta para que la Institución tenga un muy buen desempeño, y que el equipo de gestión vislumbre que, si bien cada decisión tiene como principal fundamento el bienestar Institucional, no puede estar desprovista de contenido humano y ético, teniendo en cuenta que, atrás de cada decisión, existen personas, historias, familias, proyectos, sueños, ... vida, y que esas vidas son las que dan vida a la Universidad.

En este sentido, el Dr. Kliskberg -referente en el tema a nivel nacional e internacionalexpresa que "las acciones socialmente responsables son aquellas para las cuales el resultado de la acción o beneficio que la comunidad obtiene es mayor al beneficio que obtiene la empresa" (Cammarota y Berenblum, 2009, p.58).

Este complejo entramado de acciones -que se corresponden con una gestión socialmente responsable de la Universidad- debe abordarse, como mínimo, en las siguientes dimensiones:

* La gestión interna, referida a las relaciones humanas, calidad de vida, ambiente laboral, seguridad, entre otros aspectos relacionados, principalmente, con el personal docente y no docente.

* La gestión académica o de formación, referida a los planes de estudio, contenido curricular, métodos de enseñanza y de evaluación.

* La gestión social enfocada en la relación con la sociedad y el sector privado y público.

Nos detendremos en el análisis de la dimensión de la gestión interna, y dentro de ella, en la gestión de las relaciones humanas y la calidad de vida del personal que trabaja en la Universidad porque, a mi criterio, la gestión interna impacta fuertemente en las otras dimensiones y contribuye a optimizar la performance de la Universidad en acciones de Responsabilidad Social. Para ello invitamos a algunos autores que han realizado aportes interesantes y han dado luz al tema.

"Para el mejoramiento de la calidad de vida del personal se distinguen dos aspectos básicos: el aumento de calidad de vida per se y el aumento de calidad de vida de la familia del trabajador."(Rangel, 2009, p. 22). Esto implica conocer las necesidades del ser humano, que se ponen de manifiesto en la pirámide de Abraham Maslow (Cammarota y Berenblum, 2009, p.22) quien las clasificó de la siguiente manera: 
a) Necesidades fisiológicas del ser humano, para mantener el equilibrio.

b) Necesidades de seguridad, para mantenerse protegida.

c) Necesidades de pertenencia o afiliación, relacionadas con el desarrollo afectivo del individuo.

d) Necesidades de reconocimiento, referidas a la manera en que se reconoce el trabajo del personal, se relaciona con la autoestima; estas necesidades se satisfacen cuando hay reconocimiento y gratificaciones.

e) Necesidades de autorrealización, que se hallan en la cima de la jerarquía, porque a través de su satisfacción personal encuentran un sentido a la vida mediante el desarrollo de su potencial en una actividad.

Es primordial que la organización incentive

\section{hacia el exterior acciones ya vividas en el}

ámbito interno.

El sentido de pertenencia del personal y de identidad con la Institución es clave para un clima laboral favorable, y para mejorar la calidad de vida de los trabajadores. La Responsabilidad Social podrá asimilarse a la cultura organizacional e integrarse a la gestión de la Entidad, en la medida que su público interno se sienta reconocido y valorado por ella.

El Dr. Kliskberg (Cammarota y Berenblum, 2009, p. 58) sostiene que "el equilibrio entre la vida familiar y la laboral, la motivación y la satisfacción laboral, el progreso personal y profesional, la capacitación y el entrenamiento, el buen clima laboral y el bienestar de todo el equipo de trabajo, son aspectos inherentes a la Responsabilidad Social y no deben ser descuidados... que el trabajador corre el peligro de agotamiento, que debe pensarse en su retorno para la vida de relación, mejorado, fortalecido y con mejores perspectivas para desempeñar sus otros roles".

Si centramos el análisis en el ámbito interno laboral de la Universidad, aparecen como protagonistas los docentes, no docentes y las relaciones en su ámbito de trabajo. Para un trabajador, en un sentido amplio, cuyo capital más importante es la habilidad que posee para desarrollar sus tareas, la seguridad en su empleo y las relaciones con sus compañeros son generadoras de valor, y contribuyen con la formación de su personalidad y confianza en sí mismo y en su entorno.

Trabajo y familia son los pilares de un desarrollo hacia una calidad de vida digna. Una Organización que identifica estos aspectos y que los incluye en su planificación, y entre las variables a tener en cuenta en el proceso de toma de decisiones, está influyendo y contribuyendo a una mejor calidad de vida de sus trabajadores.

Un trabajador con sobrecarga de tareas o sin reconocimiento por las actividades que realiza, no agrega valor agregado a la Institución, repercutiendo este ánimo desalentado en su ámbito familiar y en el propio laboral.

El logro de una conducta responsable interna -hacia adentro- permite proyectar un accionar socialmente responsable hacia las otras dimensiones, con una prolongación en el tiempo, en forma constante y sustentable, y no en forma aislada o periódica, para que se despliegue 
a toda la comunidad en la que está inserta.

\author{
"La Responsabilidad Social tiene la \\ característica de funcionar como un \\ bumerang, que vuelve con ímpetu al que lo \\ lanza” (Cammarota y Berenblum, 2009, p. 61).
}

\title{
3. LA RESPONSABILIDAD SOCIAL DE LA UNIVERSIDAD NACIONAL DEL NORDESTE
}

La Universidad Nacional del Nordeste -en adelante UNNE- manifiesta en su Estatuto, con firmeza y claridad, su función social y las principales acciones que se encarga de promover para su cumplimiento. Así expresa²: "La Universidad Nacional del Nordeste, comunidad espiritual de docentes, alumnos, graduados y no docentes, procura la formación integral y armónica de sus componentes, y realiza en el seno de la sociedad una labor organizada y permanente, para propender a la dignificación integral del hombre, a la formación de una conciencia democrática vigorosa y esclarecida y a la capacitación cultural y técnica de sus componentes y del pueblo de que forma parte, como órgano e instrumento de mejoramiento social, al servicio de la Nación y de los ideales de la humanidad.” Es oportuno transcribir otros párrafos incluídos en esta sección, a título ilustrativo, como forma de dimensionar la responsabilidad social asumida desde su creación:

* ...Tiene por fin primordial la generación y comunicación de conocimientos del más alto nivel, y la formación ética, cultural, técnica y profesional, contribuyendo al esclarecimiento de los problemas y necesidades de todo orden de la región, para beneficio del hombre y extendiendo su acción y servicios a la Comunidad a la que pertenece, en un clima de libertad, justicia, igualdad y solidaridad.

* Procura formar integral e interdisciplinariamente a sus componentes..., y a la vez el sentido de responsabilidad personal y social de reafirmación ético cívica... dentro de un marco de pluralidad y respetuoso disenso...

* Extiende su acción proporcionando igualdad de oportunidades a todos, ya sean estudiantes o graduados, creará las becas necesarias y todo género de ayuda que permita realizar sus estudios a quienes carezcan de medios para ello...

* Patrocina las obras de asistencia social que comprenderán en sus beneficios no solamente a los funcionarios, empleados y estudiantes de la Universidad, sino también, en cuanto fuera posible, a los asistentes y estudiosos de toda índole que se vinculen con la misma.-

* Presta servicios sociales a su personal docente y no docente, así como, eventualmente y de ser posible, a los estudiantes que cursan sus carreras en la misma. 
* Estimula toda actividad encaminada al mejoramiento de la calidad de vida, a la formación de una conciencia conservacionista del medio ambiente, al afianzamiento de las instituciones democráticas y a la reafirmación de los paradigmas de la ética, la justicia, la igualdad y la libertad.-

* La Universidad, mediante la extensión universitaria, promueve su inserción en el medio y proyección a la comunidad; participa de la responsabilidad de la labor educativa y de mejoramiento cultural del pueblo.

\section{La RS es uno de los componentes que \\ mayor impacto tiene sobre la reputación, \\ puesto que la satisfacción de las necesidades \\ legítimas de los stakeholders genera \\ relaciones basadas en la confianza, el respeto \\ y la credibilidad. (Volpentesta, 2009, p.69)}

La UNNE concreta estas manifestaciones a través del diseño e implementación de políticas de gestión acorde a los ideales con la que fue creada.

La comunidad, estudiantes, docentes, no docentes y egresados son los principales grupos de interés destinatarios de sus actividades sociales.

Si recurrimos a su historia de los últimos años ${ }^{3}$, la vida de la UNNE se distinguió por resaltar su función social en la comunidad de las provincias de Chaco y Corrientes -tal como fuera plasmado en su lema Calidad, Excelencia y Pertinencia- con una apertura nacional e internacional. El desarrollo de la pertinencia fue el camino inicial para crear una conciencia de la Responsabilidad Social en la Institución. En el año 2010 con el cambio de autoridades, se reafirmó la importancia de la UNNE como Institución social, obligando a repensar el concepto de pertinencia -bajo una mirada social y humanística- con relación al compromiso social de los universitarios y su inserción en las políticas de promoción de la inclusión social, la lucha contra la pobreza y el desarrollo local. Asimismo, se instaló la idea de que el formar profesionales significa transferir y generar capacidades y cualidades a seres humanos comprometidos con sus entornos sociales y económicos, regionales y nacionales. Esta idea se selló en el nuevo lema elegido: Gestión para la excelencia con Responsabilidad Social e Innovación, que se mantiene hasta la actualidad.

Una acción concreta de la UNNE, en esta línea, fue la creación de la Tecnicatura en Administración y Gestión de Instituciones Universitarias ${ }^{4}$-TAGIU- destinada a todo el personal No docente de las Facultades que la forman, con los siguientes objetivos:

* Formar técnicos universitarios con conocimientos teóricos y manejo de tecnologías adecuadas para administrar organizaciones universitarias.

\footnotetext{
${ }^{3}$ Con las gestiones del Rector Arq. Oscar V. Valdés (2002-2010), Dr. Adolfo Torres (2010) y el Ing. Eduardo Del Valle (actual).

${ }^{4}$ Carrera a término aprobada por Resol. № 8387/08-CD (FCE) y Resol. № 617/08-CS.
} 
* Formar técnicos universitarios capacitados para seleccionar herramientas adecuadas para efectivizar el desempeño laboral en instituciones universitarias y

* Formar técnicos universitarios para mejorar la posibilidad de movilidad intra-organizacional y de promoción escalafonaria del personal no docente de la universidad.

* Formar técnicos universitarios para administrar y optimizar la utilización de recursos humanos, financieros y de infraestructura, a fin de aumentar la eficiencia y la calidad educativa.

El título que se obtiene de Técnico en Administración y Gestión de Instituciones Universitarias acredita:

* Conocimientos y capacidades sobre administración y gestión de las instituciones universitarias.

* Conocimientos y capacidades para desenvolverse en el ámbito de trabajo universitario mediante el mejoramiento del desempeño en tareas de su competencia.

* Capacidad para asistir al personal de nivel superior (directivos y/o autoridades superiores) mediante la producción-generación de información precisa que favorezca la toma de decisiones.

* Capacidad para participar en la formulación y ejecución de políticas y estrategias propias del área en que se desempeña.

* Actitud ética y capacidad reflexiva frente a su actividad profesional y a su realidad laboral.

Entre los objetivos se pretende que los cursantes sean capaces de:

* Reflexionar sobre sus prácticas y las características positivas y negativas de sus desempeños, generando estrategias personales para la solución de situaciones reales.

* Desarrollar formas de trabajo grupal que favorezcan la comunicación, la interrelación humana y la integración de equipos.

El Programa de Formación docente continua es otra acción relevante al objetivo del desarrollo de los docentes para su crecimiento personal, profesional y con un doble beneficio porque tiene efectos directos muy positivos para con los estudiantes.

La visualización de las fortalezas y oportunidades de la implementación de acciones de Responsabilidad Social, como medio de potenciar sus objetivos, como así también la identificación de las debilidades y amenazas, permitirá a la Institución reforzar las actividades en esta materia e incluirla como tema prioritario en su agenda.

\begin{tabular}{|c|c|}
\hline $\begin{array}{l}\text { Fortalezas } \\
\text { - Crea valor } \\
\text { - Robustece la cultura organizacional } \\
\text { - Mejora la imagen y la reputación } \\
\text { - Desarrolla orgullo de pertenencia } \\
\text { - Favorece y optimiza la comunicación } \\
\text { - Profundiza las relaciones con todos los grupos de interés }\end{array}$ & $\begin{array}{l}\text { Debilidades } \\
\text { - La creencia de que con algunas } \\
\text { acciones ya está cubierta la cuota } \\
\text { de Responsabilidad Social de la } \\
\text { Organización, hasta llegar, en } \\
\text { algunos casos a confundirlas. }\end{array}$ \\
\hline $\begin{array}{l}\text { Oportunidades } \\
\text { - Representa una nueva forma de gestionar }\end{array}$ & $\begin{array}{l}\text { Amenazas } \\
\text { - Que se la utilice como una } \\
\text { herramienta de diferenciación en el } \\
\text { ámbito académico con el fin único } \\
\text { de posicionamiento }\end{array}$ \\
\hline
\end{tabular}


Es oportuno mencionar que las distintas Facultades de la UNNE apoyan fuertemente estas iniciativas y participan activamente de ellas. Por ejemplo, la Facultad de Ciencias Económicas realiza una amplia difusión de estos cursos que complementan la formación disciplinar de gran cantidad de docentes que no tienen una formación pedagógica.

La UNNE, a través de sus unidades académicas, concreta acciones de fortalecimiento de las relaciones entre los grupos interesados afectados por ella. Una de ellas, la Facultad de Ciencias Económicas, en concordancia con los valores orientadores de la Universidad a la que pertenece, tiene como misión la formación de un profesional comprometido con la Ética, la Solidaridad Social y la Responsabilidad hacia las Instituciones Republicanas. Impregnada con este espíritu de compromiso social, está abierta a desarrollar actividades que conduzcan a optimizar las relaciones internas y con los otros sectores de la sociedad, generando, al mismo tiempo, una conciencia de responsabilidad y compromiso del estudiante -futuro profesionalcon una actuación ética en la sociedad y en su práctica profesional.

Una Universidad involucrada en términos de solidaridad, valores éticos, coherencia entre el discurso y la acción, compromiso con sus trabajadores, enorgullece y penetra en la cultura de quienes trabajan en ella.

La Universidad Nacional del Nordeste está transitando el camino de la Responsabilidad Social y se proyecta en este sentido. Para recorrerlo con éxito sería beneficioso fomentar, revisar y evaluar periódicamente algunos temas prioritarios como así también orientar la gestión hacia aspectos clave que le permitan tener un comportamiento responsable en todas sus dimensiones. Se propone:

* Identificar las expectativas de los distintos grupos de interés y definir políticas acordes a ellas en búsqueda de armonizar posiciones.

* Gestionarlos efectos directos y colaterales de las acciones para minimizar las consecuencias desfavorables en los grupos interesados.

* Profundizar la comunicación con los stakeholders para confirmar acciones o reformularlas, a manera de retroalimentación en el proceso decisorio.

* Evaluar su desempeño social y medioambiental estableciendo los respectivos indicadores.

* Promover la cultura del Reporte con la finalidad de comunicar las acciones a los stakeholders.

* Incentivar las relaciones con Organizaciones del sector profesional, público y privado.

\section{CONCLUSIONES}

Las manifestaciones vertidas en este trabajo, centradas en el análisis de la importancia de la gestión interna en la Universidad Nacional del Nordeste, como una de las dimensiones de la Responsabilidad Social, con relación a uno de los grupos de interés, formado por el personal docente y no docente de la Institución, permitió arribar a las siguientes conclusiones:

* Para optimizar el desempeño en Responsabilidad Social, sería conveniente revisar y evaluar, periódicamente, las actividades relacionadas con la gestión interna. 
* Profundizar, por medio de sus prácticas, el sentido de pertenencia e identidad de su personal con la Institución y la mejora de su calidad de vida, a partir de la valoración de las tareas asignadas y de un trato justo, equitativo y solidario.

* Diversificar las acciones de Responsabilidad Social en el plan estratégico orientadas al desarrollo integral de sus stakeholders y evaluar sus impactos.

\section{PROPUESTA}

Las conclusiones arribadas permitieron proponer que sería muy beneficioso que la Universidad Nacional del Nordeste - representada en las Unidades Académicas que la formanoriente su gestión hacia aspectos que permitan abordar a la Responsabilidad Social en todas sus dimensiones, priorizando los siguientes temas en su agenda:

* Expectativas de los distintos grupos de interés: su identificación y definición de políticas acordes a ellas en búsqueda de armonizar posiciones.

* Gestión de los efectos directos y colaterales de las decisiones y actividades realizadas para minimizar las consecuencias desfavorables en los grupos interesados.

* Profundización de la comunicación con los stakeholders para confirmar acciones o reformularlas, a manera de retroalimentación en el proceso decisorio.

* Evaluación de su desempeño social y medioambiental estableciendo los respectivos indicadores.

* Promover la cultura del Reporte con la finalidad de comunicar las acciones a los stakeholders.

* Incentivar las relaciones con Organizaciones del sector profesional, público y privado.

\section{REFERENCIAS BIBLIOGRAFICAS}

Cammarota, E, Berenblum, D. (2009). Responsabilidad Social Empresarial y Gerencia Social: Partícipes necesarios para el Desarrollo Humano. En Stolar, D. y Stolar, E. (Compiladores) Responsabilidad Social Empresaria. Buenos Aires: Valletta Ediciones SRL.

Langtry, B. (1994). Stakeholders and the Moral Responsabilities of business, Business Ethics Quarterly. En Volpentesta,J.R. (2009). Gestión de la Responsabilidad Social Empresaria (431-443). Buenos Aires: Osmar D. Buyatti Librería Editorial.

Larocca, H. (2009). Responsabilidad Social Empresaria. En Saravia, F., Primavera, H., Berenblum, D., Cammarota, E., Grosso, F., Arias, G., Tarzibachi, M., Sánchez, R., Larocca, H., Marutian, J.I., Canals, M.J. y Licastro,A. Compilador: Saravia,F. ( $1^{\circ}$ Edición) Seminario de Integración y Aplicación en Proyectos Sociales (RSU) (147-162). Buenos Aires. Universidad de Buenos Aires. 
Rangel, B., Stolar, D., Camarota,E. (2009). Responsabilidad Social Empresaria. Compiladores Stolar, E. y Stolar, D. Buenos Aires: Valleta Ediciones.

Saravia, F. (2009). Responsabilidad Social Universitaria. En Saravia,F., Primavera, H., Berenblum, D., Cammarota, E., Grosso, F., Arias, G., Tarzibachi, M., Sánchez, R., Larocca, H., Marutian, J. I., Canals, M. J. y Licastro, A. Compilador: Saravia, F. ( $1^{\circ}$ Edición) Seminario de Integración y Aplicación en Proyectos Sociales (RSU) (93-113). Buenos Aires. Universidad de Buenos Aires.

Vallaeys, F. (s.f.). Trabajo La Responsabilidad Social de las Organizaciones. Obtenido el 14/04/2011 en http://www.redunirse.org/_index.php/?q=node/63

Volpentesta, J. R. (2009). Gestión de la Responsabilidad Social Empresaria,. Buenos Aires: Editorial Osmar D. Buyatti.

\section{CURRÍCULUM VITAE} ROSSANA GRECO.

Contadora Pública egresada de la Facultad de Ciencias Económicas de la Universidad Nacional del Nordeste - UNNE (1989). Especialista en Contabilidad Superior y Auditoría (2007) .

- Facultad de Ciencias Económicas. UNNE y Especialista en Docencia Universitaria (2010).

- Facultad de Humanidades. UNNE.

Docente por concurso en las Asignaturas Auditoría (desde 2003) con mayor dedicación y Estados Contables (desde 2004) con dedicación simple de la Carrera de Contador Público de la Facultad de Ciencias Económicas. UNNE. Docente en Carreras de Posgrados en la UNNE y en otras Universidades del país - Investigadora categoría 4 integrante del equipo de Investigación sobre Responsabilidad Social Empresarial con proyecto acreditado por la Secretaría General de Ciencia y Técnica de la UNNE (2009-2012). Autora y Co-autora de publicaciones en Revistas especializadas en temas de Contabilidad y Auditoría.

Egresada del V Programa de Formación de Formadores en Responsabilidad Social Empresaria (2011) organizado por la Red Iberoamericana de Universidades por la Responsabilidad Social Empresarial (REDUNIRSE).

rgreco@eco.unne.edu.ar 